\title{
Practical Planar Metric Rectification
}

\author{
Alberto Ruiz, Pedro E. López-de-Teruel and Lorenzo Fernández \\ Artificial Perception Group, University of Murcia, Spain \\ aruiz@um.es, \{pedroe,lfmaimo\}@ditec.um.es
}

\begin{abstract}
We propose a simple method for computing a metric rectification of a plane from multiple views taken by $\mathrm{K}_{i}=\operatorname{diag}\left(f_{i}, f_{i}, 1\right)$ cameras. The orthogonality properties of this camera model are exploited from an early stage to achieve a straightforward optimization process with only two degrees of freedom, even if the $f_{i}$ in all views are unknown. We study the optimization landscapes for several typical camera motions and varying amounts of image noise. We conclude that the problem is extremely ill conditioned and can only be realistically solved for rich camera motions and small amounts of image noise, preferably with at least one $f_{i}$ known in the sequence.
\end{abstract}

\section{Introduction}

The geometric constraints among multiple views of a scene can be exploited to recover a projective reconstruction. Then, affine, similar or euclidean structure can be also extracted from extravisual information or by using autocalibration techniques relying on some knowledge about the camera parameters $[3,2]$. While this general 3D reconstruction problem has been satisfactorily solved, certain applications still demand a more practical approach. In this paper we are interested in a simple and computationally efficient method for metric rectification of a single plane from several perspective images. For instance, from several aerial views of an airport we would like to obtain a map of the ground and the 3D localization of the aircraft (in 'runaway' units) (Fig. 1).

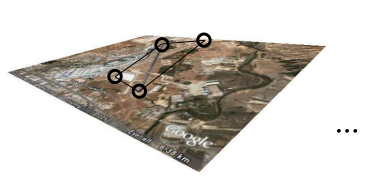

perspective 1

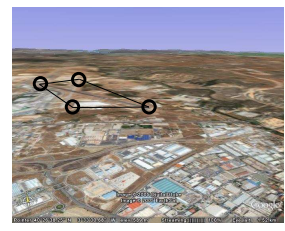

perspective $n$

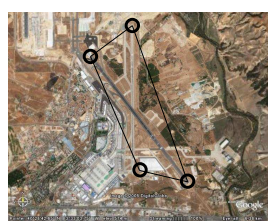

frontal

Figure 1: Hypothetical example of planar metric reconstruction.

This seems to be a basic geometric problem, apparently easier than full 3D reconstruction. As remarked in [3, 19.7], it would lead to many interesting applications, specially 
since 2D homographies are much simpler and stabler than general multiple view tensors. But, contrary to intuition, the planar case is in general more difficult. Self calibration from 3D data can be solved (in principle) by linear techniques (using e.g. the dual quadric approach [3,9]) given sufficient information about the camera parameters. In contrast, the planar case requires nonlinear optimization even from fully calibrated images. General autocalibration from planar scenes was studied by [10] and proved to be a computationally difficult nonlinear problem requiring iterative optimization methods [3]. The standard approach is based on guessing the location of the image of the the circular points of the plane in one view and transferring them to all the views to estimate the absolute conic, whose structure is constrained by knowledge about the calibration parameters.

Since it is an inherently ill-conditioned problem, we will focus on efficient, if approximate, approaches suitable to on-line work from live video sequences. As recommended by [8], we will keep the number of unknown parameters to be estimated to a minimum. We assume that the only possibly unknown camera calibration parameter is the effective focal length. This assumption is approximately valid in a wide range of modern commercial cameras and it is customarily adopted in most geometric vision applications designed to work with live video sequences [1]. Deviations from this model, e.g., radial distortion or special image aspect ratio, should be corrected in advance.

We will propose a method for planar metric reconstruction which extensively exploits the orthogonality constraints supplied by this camera model.

\section{Problem setting}

Consider a sequence of $n$ images $S=\left\{s_{1}, s_{2}, \ldots, s_{n}\right\}$ of a plane $\pi$ taken from a freely moving and possibly zooming camera. Let $\mathrm{H}_{i, j}$ be the homography from view $s_{j}$ to view $s_{i}$. The 'interimage' homographies $\mathrm{H}_{i, j}$ are not independent from each other: for any $k$, $\mathrm{H}_{i, j}=\mathrm{H}_{i, k} \mathrm{H}_{k, j}$ (see also the concept of superhomography in [6]). We assume that certain geometric features can be identified in the images in such a way that any $\mathrm{H}_{i, j}$ can be computed. Typically we derive them from a chain of homographies $\mathrm{H}_{k+1, k}$ relating consecutive images in the sequence. We will consider first the simplest case, in which the available homographies are essentially correct. Section 4 studies the effects of noise.

The camera matrix of the $i$-th image can be written as $\mathrm{P}_{i}=\mathrm{K}_{i}\left[\mathrm{R}_{i} \mid-\mathrm{R}_{i} C_{i}\right]$, where $\mathrm{K}_{i}=\operatorname{diag}\left(f_{i}, f_{i}, 1\right)$ and $\mathrm{R}_{i}$ and $C_{i}$ are the orientation and localization of the camera. For convenience, we choose a 3D euclidean frame in which $\pi=(0,0,1,0)$ is the ground plane $(z=0)$. Then the homography from $\pi$ to $s_{i}$ is just $C_{i}=\tilde{\mathrm{P}}_{i}=\mathrm{K}_{i}\left[\tilde{\mathrm{R}}_{i} \mid-\mathrm{R}_{i} C_{i}\right]$, where $\tilde{\mathrm{M}}$ denotes $\mathrm{M}$ with the third column removed. In principle, we can express $\mathrm{H}_{i, j}=\mathrm{C}_{i} \mathrm{C}_{j}^{-1}$ explicitly in terms of the ingredients in $\mathrm{P}_{i}$ and $\mathrm{P}_{j}$, but the expression is not particularly useful for our present purposes. Our goal is to synthesize a frontal view of the plane, denoted by $s_{0}$, as well as the camera matrices $\mathrm{P}_{i}$ from the interimage homographies $\mathrm{H}_{i, j}$. Therefore, we must find a rectifying homography, denoted by $\mathrm{C}^{-1}$, which transforms some view, say $s_{1}$, to the frontal, metrically rectified $s_{0}$. Its inverse, $\mathrm{C}$, is closely related to the camera homography of the first view $\mathrm{C}_{1}: \mathrm{C}_{1}=\mathrm{CS}$, where $\mathrm{S}$ is some inessential similar transformation depending on the arbitrarily chosen coordinate systems. The four essential degrees of freedom in $\mathrm{C}$, associated to the circular points, must be discovered from a more or less ingenious analysis of the interimage homographies.

There is a crucial difference between the unknown camera homographies $\mathrm{C}_{k}$ and 
the interimage homographies $\mathrm{H}_{i, j}$. The camera homographies have an internal structure caused by the internal rotation $\tilde{\mathrm{R}}_{i}$, and this structure is (at least in principle) detectable. For instance, any camera homography $\mathrm{C}$ with arbitrary calibration matrix $\mathrm{K}$ verifies the following standard orthogonality constraint:

$$
\mathrm{C}^{\top} \omega \mathrm{C}=\left[\begin{array}{ccc}
v & 0 & - \\
0 & v & - \\
- & - & -
\end{array}\right]
$$

where $\omega=\mathrm{K}^{-T} \mathrm{~K}^{-1}$ is the image of the absolute conic. (In some cases $\omega$ can be easily deduced from $C$.)

In contrast, the individual interimage homographies lack such characteristic structure. However, the whole set of interimage homographies does still have some structure, that could be exploited to obtain the desired frontal view. The key idea is that $s_{0}$ is a frontal view consistent with the set $\mathrm{H}_{i, j}$ if there is some rectifier $\mathrm{C}^{-1}$ such as all the $\mathrm{C}_{i}=\mathrm{H}_{i, 1} \mathrm{C}$ become camera homographies verifying property (1).

There is a direct analogy between the self-calibration techniques based on the absolute dual quadric and the metric rectification of a plane based on interimage homographies. In the first case the multiview tensors provide a set of projective cameras $M_{i}$ and structure $X_{i}$ consistent with the image $s_{i}$ such as $s_{i}=\mathrm{M}_{i} \mathrm{X}_{i}$. There is a 3D projective ambiguity A since $s_{i}=\left(\mathrm{M}_{i} \mathrm{~A}_{i}\right)\left(\mathrm{A}_{i}^{-1} \mathrm{X}_{i}\right)$; we must find the rectifier A which produces $\mathrm{M}_{i} \mathrm{~A}_{i}$ verifying the calibration constraints. For planes we have a similar situation, with the difference that the projective reconstruction is directly available as any of the views, e.g. $s_{1}: s_{i}=\mathrm{H}_{i, 1} s_{1}$. There is a projective ambiguity $s_{i}=\left(\mathrm{H}_{i, 1} \mathrm{C}\right)\left(\mathrm{C}^{-1} s_{1}\right)$ and we are interested in the camera homography $\mathrm{C}$ of the first image, which produces $\mathrm{H}_{i, 1} \mathrm{C}$ verifying the properties of camera homographies with the assumed calibration constraints (Fig. 2).

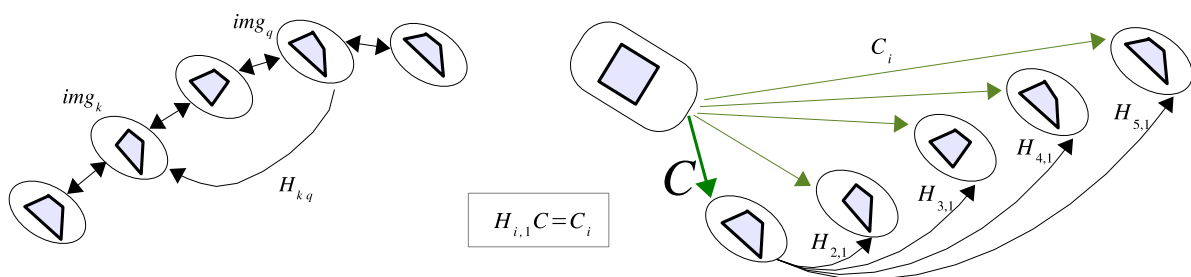

Figure 2: The solution C converts the interimage homographies into cameras.

The rectifier $\mathrm{C}^{-1}$ can be expressed as a conjugate rotation $\mathrm{KRK}^{-1}$ synthesizing a frontoparallel view, which in the particular case of a $\mathrm{K}=\operatorname{diag}(f, f, 1)$ camera can be explicitly written as:

$$
\mathrm{C}\left(l_{\infty}^{\prime}, f\right)=\left[\begin{array}{ccc}
\cos \rho & -\cos \alpha \sin \rho & -f \sin \alpha \sin \rho \\
\sin \rho & \cos \alpha \cos \rho & f \cos \rho \sin \alpha \\
0 & -\sin \alpha / f & \cos \alpha
\end{array}\right]
$$

where $\rho$ is the roll angle and $\alpha=\arctan (h / f)$ is the tilt angle of the camera, and $h$ is the height of the horizon $l_{\infty}^{\prime}=(\cos \rho, \sin \rho,-h)^{\top}$. 


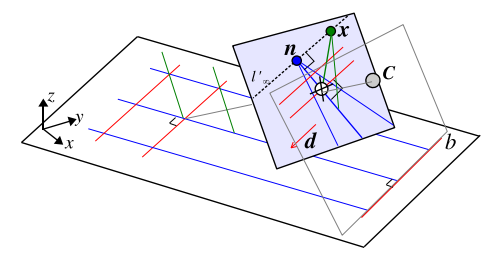

Figure 3: Geometric construction for $f$ in a $\operatorname{diag}(f, f, 1)$ camera.

\section{Practical planar rectification}

In this section we describe a remarkably simple method to find $\mathrm{C}$ which exploits the orthogonality properties of the $\operatorname{diag}(f, f, 1)$ camera model to achieve a straightforward $2 \mathrm{D}$ optimization process. We will describe the method for increasing levels of difficulty.

\subsection{Full calibrated images}

Consider first the easiest situation, in which all the $f_{i}$ in the image sequence are known. There are only two degrees of freedom in the rectifying homography, namely the location $(\rho, h)$ of the horizon $l_{\infty}^{\prime}$. We can minimize the average violation cost of the property (1) over all the tentatively corrected homographies $C_{k}=\mathrm{H}_{1, k} \mathrm{C}(\rho, h)$. The contribution of $\mathrm{C}_{k}^{\top} \omega_{k} \mathrm{C}_{k}=\left\{a_{i, j}\right\}$ can be measured by $\left(a_{1,2} / a_{1,1}\right)^{2}+\left(a_{2,2} / a_{1,1}-1\right)^{2}$. Section 4 shows the aspect of this optimization surface for a number of image sequences. In general it is reasonably well behaved and any conventional convex minimization algorithm will be able to obtain an acceptable solution with small computational effort.

\subsection{Known $f_{1}$}

The rectifying homography (2) only depends on the calibration parameter $f_{1}$ of the first image. If it is known, we can still compute a 2-d.o.f error surface, depending on the position of the horizon, even if the rest of the $f_{i}$ are unknown. The reason is that in the case of a $\operatorname{diag}(f, f, 1)$ camera the effective focal $f$, required to compute the cost via $\omega=\operatorname{diag}\left(1,1, f^{2}\right)$, can be easily extracted [3, ch.8] from the tentatively corrected homographies plane-image $\mathrm{C}_{k}$. In this work we use a simple geometric construction (Fig. 3) based on vanishing points separated 45 degrees:

$$
\begin{gathered}
f=\sqrt{\|x-n\|^{2}-\|n\|^{2}} \\
n=\mathrm{CSC}^{\top} l_{\infty} \quad x=\mathrm{CFC}^{\top} l_{\infty} \quad l_{\infty}=(0,0,1)^{\top} \quad \mathrm{S}=\left[\begin{array}{lll}
1 & 0 & 0 \\
0 & 1 & 0 \\
0 & 0 & 0
\end{array}\right] \quad \mathrm{F}=\left[\begin{array}{ccc}
1 & 1 & 0 \\
-1 & 1 & 0 \\
0 & 0 & 0
\end{array}\right]
\end{gathered}
$$

In a frontal view $f$ cannot be extracted, but in this case the consistency function can be based in the deviation of the tentative camera from a similar transformation.

Clearly, if the horizon is wrong the interimage homographies will in general not be appropriately corrected, producing some consistency error. When they are correctly converted into cameras by the right horizon the estimated $f_{i}$ will be also correct. In section 4 
we will show the aspect of this type of error surface for different planar rectification image sequences. The solution is now enclosed in a flatter region, since, informally speaking, there are additional candidate horizons generating true cameras with wrong $f_{i}$ but which should be admitted as possible solutions.

\subsection{All $f_{i}$ unknown}

The most interesting situation arises when all the effective focal lengths $f_{i}$ are unknown. Since the rectifier depends on the unknown $f_{1}$, in principle the error surface seems to have 3 d.o.f (the horizon and $f_{1}$ ). However, thanks to the orthogonality properties of the $\operatorname{diag}(f, f, 1)$ cameras, we can still build a 2-d.o.f. error surface. In this kind of cameras the horizon of a plane induces the view of a right angle 'for free' (Fig. 4(a)). Therefore, given a tentative horizon in the first view, we can transfer it to the rest of views using the interimage homographies and reproject back a number of right angles to the first view in such a way that a estimate of $f_{1}$ can be easily achieved (Fig. 4(b)).

The estimation of $f$ from right angles could of course be performed by the standard method based on the conic dual to the circular points, which requires two right angles if the horizon is known [3, ch.2]. Alternatively, we can take advantage of the camera model $\mathrm{K}=\operatorname{diag}(f, f, 1)$ from the start, and obtain $f$ in closed form from a single right angle using geometric arguments (Fig. 4(c)). (This is a particular case of the more general expression given in [5, p.105].) This procedure is significantly simpler and faster. ${ }^{1}$

In summary, using $\operatorname{diag}\left(f_{i}, f_{i}, 1\right)$ cameras, a metric rectification can be automatically obtained from an affine rectification (and the interimage homographies); we only need the horizon of the plane. It is again a 2-d.o.f. optimization problem, remarkably simpler than searching for the circular points (4-d.o.f.). Section 4 shows this kind of error surface. Since now all the $f_{i}$ are estimated from the tentative camera homographies, the solutions are enclosed in even flatter regions, suggesting that optimization will only be successful in low noise situations.

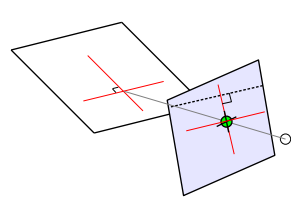

(a)

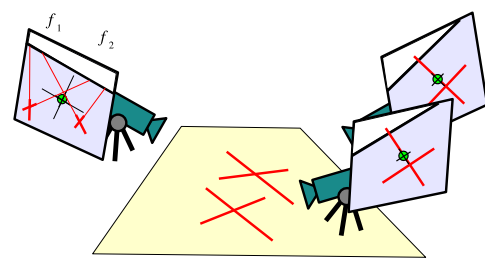

(b)

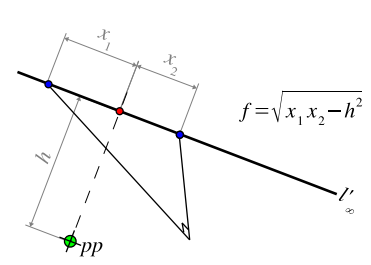

(c)

Figure 4: (a) The image of a right angle induced by the horizon in a $\operatorname{diag}(f, f, 1)$ camera. (b-c) Right angles induced by a tentative horizon provide estimates of $f_{1}$.

\footnotetext{
${ }^{1}$ In noiseless situations if the $f_{1}$ estimates produced by all the additional views are different then the tentative horizon is wrong. In principle, this might be used for a more efficient optimization than eq. (1). Unfortunately the dispersion in the estimates caused by image noise cannot be easily distinguished from a wrong horizon.
} 


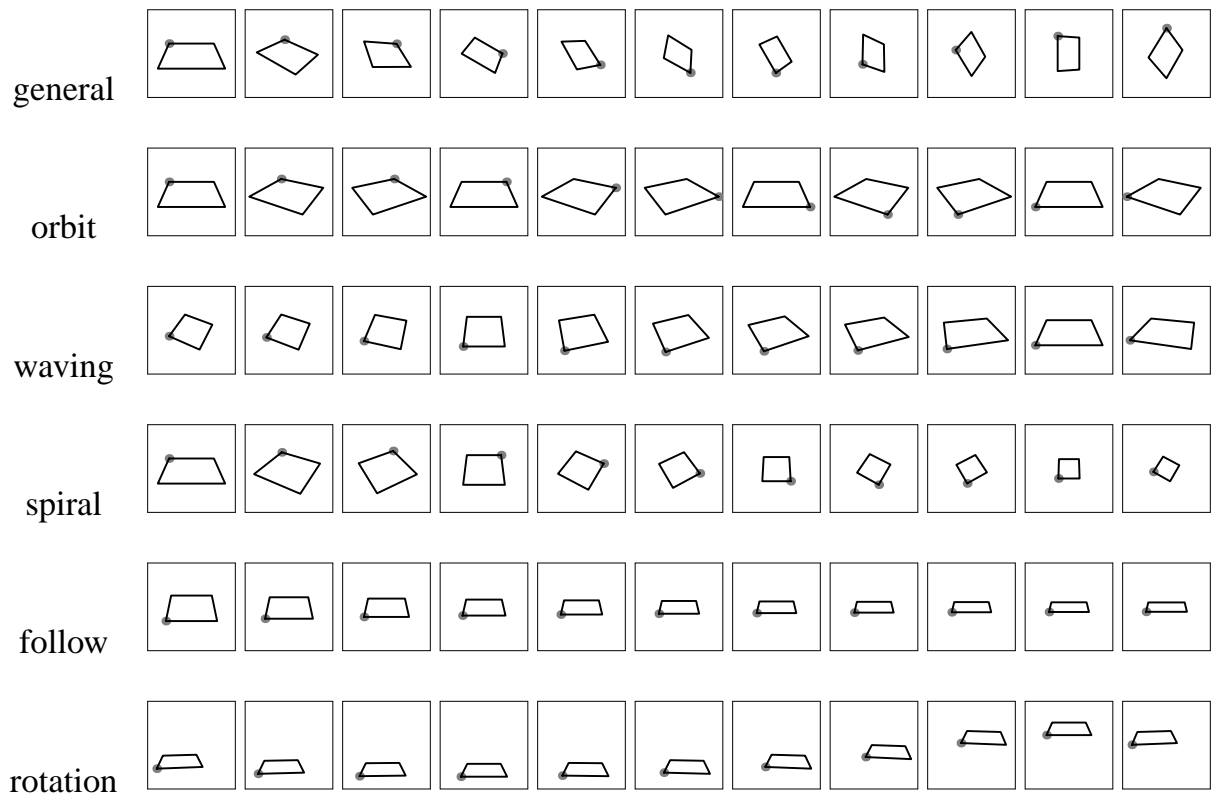

Figure 5: Camera motions used in the experiments.

\subsection{Constant $f$}

Finally, if it is known that the $f_{i}$ are all equal to some unknown parameter $f$ then we can estimate it by the procedure explained above, and improve the error surface using the estimated $f$ for computing the consistency property, instead of the general procedure in Sect. 3.2. Sect. 4 also shows the error surface in this case.

\section{Experiments}

We have evaluated the four variants of the proposed method in several typical camera motions (Fig. 5). We show the error surfaces induced by the position of the horizon (Fig. 6) and the corresponding rectified reference square (Fig. 7) under different circumstances:

A) Noiseless interimage homographies (first row in Fig. 6).

B) Small amount of image noise. The interimage homographies have been estimated from images of a reference unit square whose vertices have been contaminated with a random value uniformly distributed between -0.01 and 0.01 , corresponding to $1 \%$ of the image resolution. ${ }^{2}$ There are 11 views (second row in Fig. 6).

C) Large noise, $5 \%$ of the image resolution and 11 views (third row).

D) Again 5\% noise but in this case there are 51 views (fourth row).

In all cases the error surface has a $100 \times 100$ resolution, with roll angle from $-60^{\circ}$ to

\footnotetext{
${ }^{2}$ In real applications we may have more than four points available to compute the interimage homographies. In that case the precision in the estimations will be higher for the same amount of noise, and also global constraints ([6]) can be exploited. Therefore, our results must be interpreted as a worst case situation.
} 
$+60^{\circ}$ in the vertical axis and horizon height from 0 to 3 in the horizontal axis (pixel range is $(-1,1)))$. The level curves and colors in the error surfaces represent the same scale in all experiments, ranging from zero cost (white) to 5 normalized units (black). The position of the true horizon is marked with a red dot.

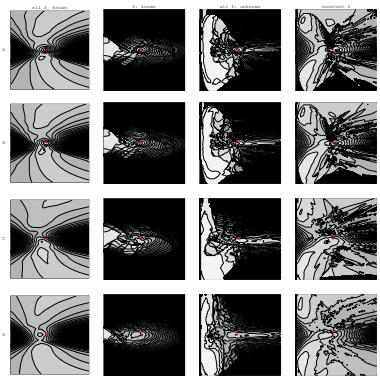

a) general motion

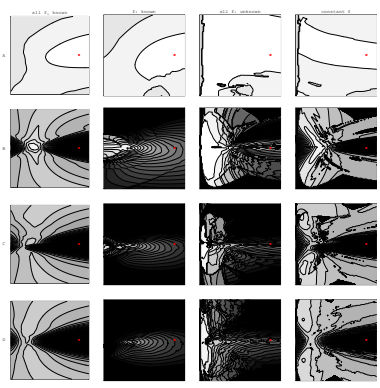

d) spiral

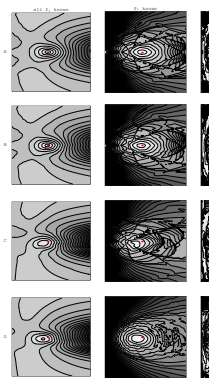

b) orbit
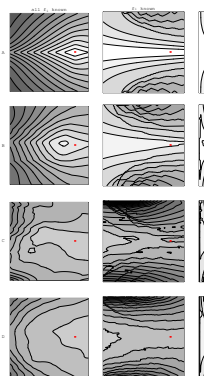

e) follow

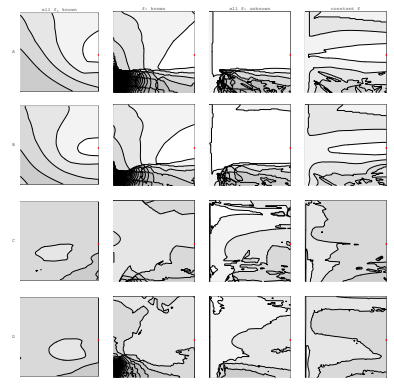

c) waving

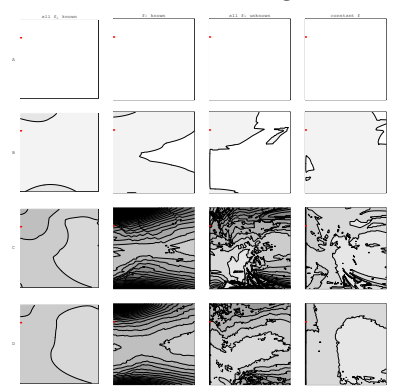

f) rotation

Figure 6: Error surfaces (horizon consistency) for the six image sequences studied. The columns in each $4 \times 4$ group of experiments correspond respectively to procedures 3.1 , 3.2, 3.3, and 3.4, and the rows correspond to the noise amounts A, B, C, and D described above. This figure is better appreciated by zooming in the electronic version of the paper.

General motion. In the first image sequence (first row in Fig. 5) the camera turns around the reference object increasing height, distance, roll angle, and $f$. The optimization surfaces of the four proposed rectification procedures are shown in Fig. 6(a). This situation is easily solvable in the full calibrated case (first column). Small amounts of noise are tolerable (B), and large amounts of noise could be removed by taking more images (D). If only $f_{1}$ is known (second column), the problem is still solvable, although the optimization would probably need several starting points. When all the $f_{i}$ are unknown the error surface becomes much more complicated, suggesting that this situation is very difficult to solve in practice. The last column is not applicable here (the $f_{i}$ are not constant in the sequence). Fig. 7(a) shows the rectified reference square using the best solution found in the explored horizon range for the noisy experiments (rows B,C, and, D).

Orbit. In this sequence the camera rotates around the object at a fixed distance and height, with fixed $f$. The error surfaces are shown in Fig. 6(b). This sequence seems to 


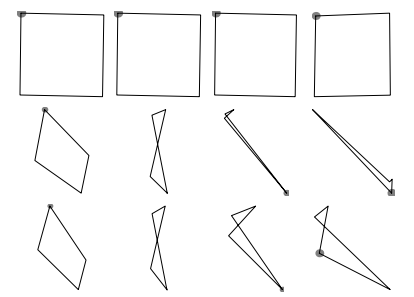

a) general motion

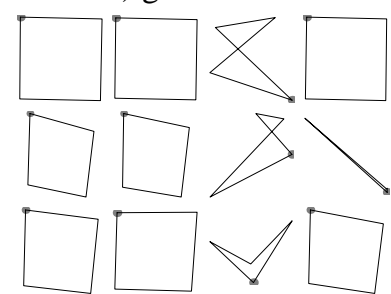

d) spiral

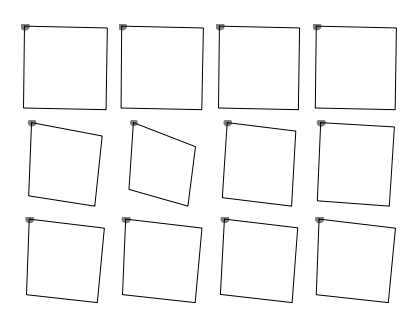

b) orbit

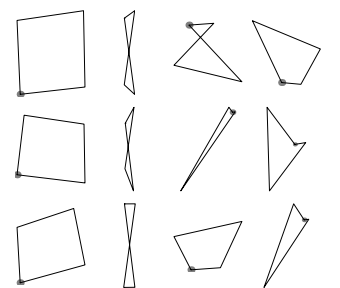

e) follow

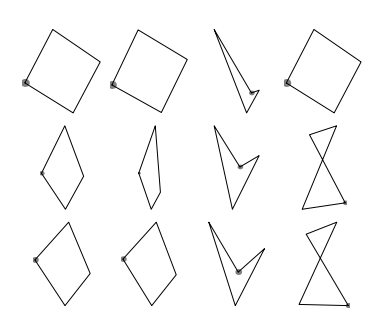

c) waving

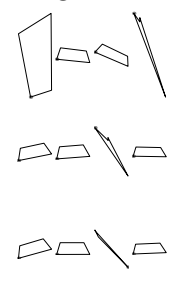

f) rotation

Figure 7: Rectified object for the noisy cases (B,C, and D) in Fig. 6

be solvable: the error surfaces are smooth in the full calibrated case and with $f_{1}$ known, and can also be solved in the uncalibrated case even with appreciable amounts of noise. The rectified references are shown in Fig. 7(b).

Waving. The camera moves laterally and changing height, with fixed $f$. The error surfaces are shown in Fig. 6(c). This problem is significantly more difficult (Fig. 7(c)). Probably because the object is seen only from one side the error surfaces are very flat, and only small amounts of noise are hardly tolerable in the full calibrated case. Even with noiseless images the uncalibrated situation is essentially unsolvable.

Spiral. The camera rotates around the object, increasing height, with fixed $f$. This sequence is difficult but could be solved with known $f_{i}$ or at least $f_{1}$ (Fig. 6(d), Fig. 7(d)).

Following. The camera follows an object which is moving away, increasing $f$ to get a similar apparent size. The error surfaces are shown in Fig. 6(e). This sequence is extremely difficult (Fig. 7(e)) and could only be solved in the full calibrated case (estimation of $f_{1}$ requires changes in the principal ray) with very small amounts of noise.

Rotation. In this sequence the camera follows the surroundings of the reference object from a common projection center, with fixed $f$. This camera motion is too restricted, so the problem is of course unsolvable (Fig. 6(f) and Fig. 7(f)).

In practical applications we optimize the starting point obtained from a more coarse exploration of the optimization surface. Using the Nelder-Mead Simplex algorithm in GSL [7] we obtain computation times of order of the second in a conventional machine.

\subsection{Planar motion}

If camera motion can be controlled then linear solutions can be often achieved [4]. Interestingly, for pure planar motion with constant internal parameters the problem has zero(!) degrees of freedom. In this case the horizon of the plane and the image of the circular 
points are fixed (modulo noise) in the sequence. This is an important situation in which we can easily cope with image noise simply by taking a larger number of views.

The image of the circular points for $\mathrm{K}=\operatorname{diag}(f, f, 1)$ and tilt $\alpha$ (with roll $\rho=0$ ) are:

$$
I^{\prime}=(-i f \sec \alpha, f \tan \alpha, 1)^{\top}, \quad J^{\prime}=\overline{I^{\prime}}
$$

so $l_{\infty}^{\prime}, f$, and the rectifier (eq. 2), can be immediately extracted from the fixed points:

$$
n=\frac{I^{\prime}+J^{\prime}}{2}, \quad \rho=\tan ^{-1} \frac{n_{y}}{n_{x}}, \quad\|n\|=f \tan \alpha, \quad\left\|I^{\prime}-J^{\prime}\right\|=2 f \sec \alpha
$$

where the point $n$, which univocally specifies the horizon for this camera model, is the vanishing point of lines in the plane which are parallel to the optical axis (eq. 3, Fig. 3).

The estimation of the fixed point $I^{\prime}$ from noisy homographies can be approached from different viewpoints. A first idea is finding the minimun squared error (MSE) solution to the null space of the block matrix containing all the $\mathrm{H}_{i j}-\lambda_{i j} \mathrm{I}$, where $\lambda_{i j}$ is the appropriate complex eigenvalue of $\mathrm{H}_{i j}$. This provides a initial estimate of $I^{\prime}$ that could be iteratively refined by recomputing the eigenvalues $\lambda_{i j}$ and the MSE null space of the updated block matrix. A simpler alternative is some kind of average of the fixed points generated by the available homographies using a robust multidimensional location estimator.

Fig. 8(a) shows the fixed points of independent noisy interimage homographies $\mathrm{H}_{1, k}$ of a planar motion sequence, represented as segments (to account for the 4 d.o.f. of a complex point). Fig. 8(b) contains the fixed points of the whole set $\mathrm{H}_{i, j}$. The true fixed point is represented in blue and the robust estimation in red. Fig. 8(c) shows the views transferred to the first one, corrected by the estimated roll, tilt and $f$ (otherwise all transferred views will be coincident). These views can now be averaged out (using again a robust estimator) and rectified, obtaining a very precise reference square (Fig. 8(d)).

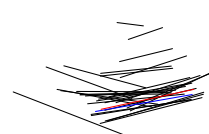

(a)

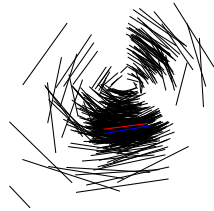

(b)

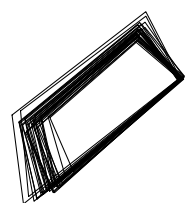

(c)

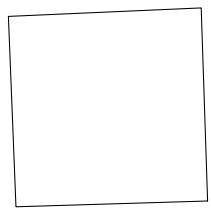

(d)

Figure 8: (a)-(b) Robust estimation of one of the circular points. (c) Corrected views transferred to the first view. (d) Robust estimation of the common view, rectified.

\section{Conclusion}

This paper proposes a simple 2-dimensional optimization procedure to obtain a metric rectification of a single plane from multiple views which exploits the orthogonality properties of the $\mathrm{K}=\operatorname{diag}(f, f, 1)$ camera model. Using this kind of camera we can obtain the desired metric rectification just from the location of the horizon, since the interimage homographies induce estimations of the $f$ parameter required to synthesize the appropriate 
rectifying conjugate rotation. The images of the circular points are attached to predictable positions in the horizon. Therefore, we can estimate the horizon by minimization of a reasonably simple cost function based on the consistency of the recovered cameras, even if all the $f_{i}$ parameters are initially unknown.

Unfortunately, this problem is extremely ill-conditioned, even for this simplified camera model. The $2 \mathrm{D}$ nature of the consistency function allows visual inspection of the problem complexity, so we have computed a large region of the optimization surfaces for a number of typical camera motions that could be used in practical applications. We conclude that in most cases this kind of automatic planar metric rectification can only be achieved for rich camera motions and low image noise, preferably with some $f_{i}$ known.

Future work includes a better parameterization of the horizon. If $f_{1}$ is known we can express it in terms of the roll and tilt angles of the camera. In any case, some nonlinear spacing of the horizon height must be studied. On the other hand, the rectified view should be computed by taking into account not only the minimum cost rectification parameters $(\rho, y, f)$, but also a corrected version of the homographies, as in the pure planar case. Finally we are developing the required programming infrastructure to allow some limited form of real-time planar metric rectification from live image sequences.

\section{Acknowledgments}

The authors would like to thank the anonymous reviewers for their suggestions. This work has been supported by the Spanish MCYT grant TIC2003-08154-C06-03.

\section{References}

[1] A. J. Davison and D. Murray. Simultaneous localisation and map-building using active vision. IEEE Trans. Pattern Analysis and Machine Intelligence, July 2002.

[2] O. Faugeras, Q.-T. Luong, and T. Papadopoulo. The Geometry of Multiple Images. MIT Press, 2001.

[3] R. Hartley and A. Zisserman. Multiple View Geometry in Computer Vision. Cambridge University Press, 2 edition, 2004.

[4] J. Knight, A. Zisserman, and I. Reid. Linear auto-calibration for ground plane motion. In CVPR'03, 2003.

[5] P.E. López-de-Teruel. PhD thesis, (in spanish) DITEC, University of Murcia, Spain, 2003. Available in http://ditec.um.es/ pedroe/documentos/Tesis.pdf.

[6] E. Malis and R. Cipolla. Camera self-calibration from unknown planar structures enforcing the multiview constraints between collineations. PAMI, 24(9), Sept. 2002.

[7] GNU. Scientific library, http://www.gnu.org/software/gsl.

[8] L. Quan and W. Triggs. A unification of autocalibration methods. In ACCV, 2000.

[9] W. Triggs. Auto-calibration and the absolute quadric. In Proc. CVPR, 1997.

[10] W. Triggs. Autocalibration from planar scenes. In Proc. ECCV’98, 1998. 